\title{
Simple Spectrophotometric Sequential Injection Analysis System for Determination of Serum Calcium
}

\author{
Yuwadee Boonyasit $^{1}$, Chakorn Chinvongamorn ${ }^{2}$, Orawon Chailapakul ${ }^{3}$, Wanida Laiwattanapaisal $^{4}$ \\ ${ }^{1}$ Graduate Program in Clinical Biochemistry and Molecular Medicine, Faculty of Allied Health Sciences, \\ Chulalongkorn University, Bangkok, Thailand \\ ${ }^{2}$ Department of Chemistry, Faculty of Science and Technology, Rajamangala University \\ of Technology Thanyaburi, Pathum Thani, Thailand \\ ${ }^{3}$ Sensor Research Unit, Department of Chemistry, Faculty of Science, Chulalongkorn University, Bangkok, Thailand \\ ${ }^{4}$ Department of Clinical Chemistry, Faculty of Allied Health Sciences, Chulalongkorn University, Bangkok, Thailand \\ Email:wanida.k@chula.ac.th
}

Received November 15, 2011; revised December 21, 2011; accepted January 2, 2012

\begin{abstract}
A sequential injection analysis (SIA) system for the determination of total calcium in blood has been implemented. The assay principle is based on the arsenazo III method, in which the indicator's complex with calcium forms a blue-purple colour. The absorbance peak height at $650 \mathrm{~nm}$ was proportional to the calcium content in samples. A linear range was obtained from $2.5-15 \mathrm{mg} \cdot \mathrm{dL}^{-1}\left(\mathrm{r}^{2}=0.999\right)$, with a detection limit of $0.430 \mathrm{mg} \cdot \mathrm{dL}^{-1}(3 \sigma)$. Magnesium, glucose, haemoglobin, and bilirubin at $123,1,000,50$, and $0.5 \mathrm{mg} \cdot \mathrm{dL}^{-1}$, respectively, were not found to interfere with the proposed system significantly (recovery $99.1 \%-104.4 \%$ ). The sampling frequency was $30 \mathrm{~h}^{-1}$, in which the carry-over effect was negligible $(0.69 \%)$. The proposed SIA system was successfully applied to the determination of calcium from serum samples $\left(r^{2}=0.970 ; n=30\right)$.
\end{abstract}

Keywords: Sequential Injection Analysis System; Calcium; Arsenazo III; Green Chemistry; Spectrophotometry; Clinical Analysis

\section{Introduction}

Blood calcium is distributed among several forms, of which the major forms are free calcium ions and proteinbound calcium. Calcium content is closely associated with a large number of diseases, such as hypo-and hyperthyroidism, acute pancreatitis, malignancy, and vitamin $\mathrm{D}$ deficiency [1]. Thus, many researchers have turned their attention to developing quantitative measurements of calcium levels in serum specimens because of the potential for diagnosing these disorders. Until recently, improvements in alternative approaches for assaying calcium depended heavily on the types of calcium found in blood circulation.

The use of ionised calcium, a useful marker for assessing calcium regulation disorders, makes the accurate interpretation of results difficult due to the effects from maintaining $\mathrm{pH}$ levels that rely on the carbon dioxide content of the samples [2]. In contrast, the determination of total calcium ions is more popular and widely used in most clinical laboratories because of its simplicity of operation. The methods based on dye-binding, such as o-cresolphthalein complexone and arsenazo III, are commonly used for determining total blood calcium. Consid- ering a dye that specifically forms a complex with calcium, arsenazo III can be simply prepared and used without the addition of 8-hydroxyquinoline, a potential magnesium masking reagent [3]. It offers an advantage over other dye-binding methods that require a separate reagent preparation.

Currently, numerous approaches for quantifying the amount of calcium based on arsenazo III have been reported, such as the spectrophotometric method $[3,4]$, microfluidic-based platforms [5-7], flow injection analysis [8-10], and sequential injection analysis (SIA) [11,12]. The preceding studies on the determination of calcium by means of SIA system have been applied and validated, but solely for the environmental samples, no utility being expressed for the complex biological samples. In regard to clinical assessment, the ability to make rapid measurements has become the cornerstone of serum calcium assays. Owing to an anaerobic metabolism of blood cells, the specimens should be handled and analysed promptly. The alteration of sample $\mathrm{pH}$ can affect calcium levels; therefore, an analysis should be completed within 1 hour [2]. Typically, turnaround time is of considerable importance in batch processing, thereby utilizing SIA is an alternative approach to analytical systems. With the proposed SIA 
technique, it is well-known in terms of its fully automatic operation, simple sampling procedure, high throughput of samples, low reagent consumption, inexpensive instrumentation, and short analysis time [13]. Unless otherwise stated, SIA is appropriate for the overwhelming majority of clinical laboratories for monitoring calcium status at a patient's bedside due to its fast processing and great precision.

Although calcium quantification using SIA has previously been reported in conjunction with the o-cresolphthalein complexone reaction [14-16], the use of arsenazo III for serum calcium determination has yet to be investigated. For this reason, we proposed here a simple, rapid, and greener analytical procedure for real sample analysis of total serum calcium. Using this configuration of SIA, we obtained excellent accuracy and reproducibility for this calcium assay.

\section{Materials and Methods}

\subsection{Reagents and Chemicals}

Analytical grade chemicals were used in this study. For the calcium standard, calcium carbonate was supplied by Mallinckrodt (St. Louis, USA). Arsenazo III and bilirubin were purchased from Fluka (Buchs, Switzerland). Piperazine-N,N'-bis[2-ethanesulfonic acid (PIPES), 2-(N-morpholino)ethanesulphonic acid (MES), haemoglobin, magnesium sulphate, and D-glucose were acquired from Sigma (St. Louis, USA). Hydrochloric acid was purchased from Merck (Darmstadt, Germany). Taking into account method validation, calcium assay kits from Randox Laboratories (Crumlin, United Kingdom) were used for a comparison study. To obtain varying calcium levels in samples, two levels of control serums (Liquid Assayed Multiqual ${ }^{\circledR}$ ) from Bio-Rad Laboratories (Berkeley, USA) were used to spike in real serum samples.

For the preparation of calcium stock standards (200 $\mathrm{mg} \cdot \mathrm{dL}^{-1}$ ), five grams of dried calcium carbonate were dissolved in $1 \mathrm{M}$ hydrochloric acid and diluted to $1 \mathrm{~L}$ with Milli-Q water (Millipore, USA).

\subsection{Apparatus}

The proposed system ensured the configuration of the SIA method, including a syringe pump (Cavro XL 3000, Cavro Scientific Instruments Inc., USA) equipped with a $2.5 \mathrm{~mL}$ syringe, a multi-position selection valve (Cavro Smart Valve, Cavro Scientific Instruments Inc., USA), and a spectrophotometric detector (Evolution 600, Thermo Scientific, USA) incorporated with an $18 \mu \mathrm{L}$ quartz flowthrough cell. The system is depicted in Figure 1. Each absorbance peak height was recorded and assessed with commercial software. PTFE tubing with $0.8 \mathrm{~mm}$ i.d. (Cole-Palmer Instrument Company, USA) was used to connect the different components of the flow system, and all connectors used were made of PEEK (Upchurch Scientific, USA).

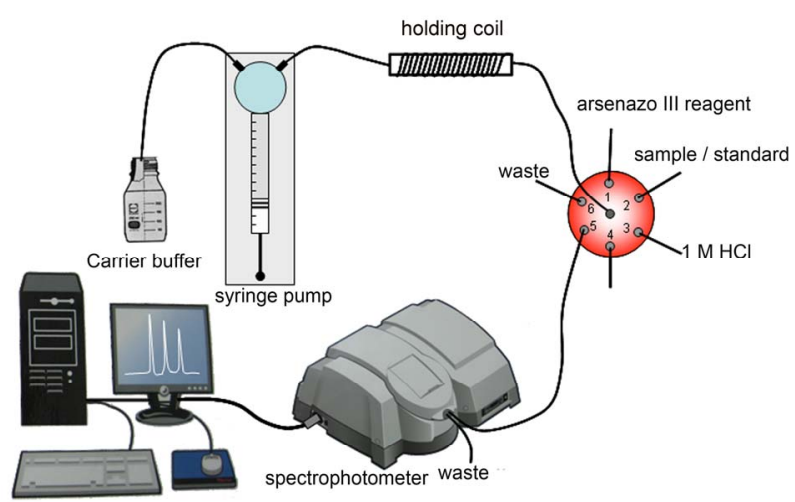

Figure 1. Set-up of SIA calcium assay system based on arsenazo III method.

The conventional arsenazo III, a batch cuvette-based method, was performed using the same UV-VIS spectrophotometer mentioned above, according to the protocol described in the instruction manual provided by Randox Laboratories.

\subsection{Procedure}

Unless otherwise stated, the whole operating sequence of the SIA system for quantitative calcium analysis is revealed in Table 1. The system began by aspirating the carrier buffer into syringe. The arsenazo III and standard/sample solutions then followed the carrier buffer into the holding coil, and the arsenazo III reagent was subsequently aspirated again. The flow direction was then reversed, resulting in the displacement of the reaction mixture to the flow-through cuvette. Using the spectrometric detector, the chemical reaction between arsenazo III and calcium was quantified at $650 \mathrm{~nm}$ as a result of its blue-purple complex. To avoid contamination from other substances, a solution of $1 \mathrm{M}$ hydrochloric acid was injected into the SIA system and subsequently washed with the carrier buffer.

\section{Results and Discussion}

\subsection{Optimum Performance of Calcium Assay}

\subsubsection{Operation Sequence}

Preliminary results demonstrated that $200 \mu \mathrm{L}$ arsenazo III reagent was particularly suitable for interacting with $10 \mu \mathrm{L}$ calcium. Thus, we intended to select a reagent/ sample ratio of 200:10. According to Figure 2(a), the aspiration sequence was optimised by studying four variants of the aspiration sequence. The operation sequence 2 (SQ-2) gave the highest analytical signal when evaluated with 5 and $15 \mathrm{mg} \cdot \mathrm{dL}^{-1}$ calcium, as shown in Figure 2(b). For this reason, the aspiration sequence of arsenazo III reagent $(100 \mu \mathrm{L})$, sample $(10 \mu \mathrm{L})$, and arsenazo III reagent $(100 \mu \mathrm{L})$ was selected for further studies. 
Table 1. Operation sequence of SIA system for serum calcium determination.

\begin{tabular}{|c|c|c|c|c|c|}
\hline Step & Operation & Valve of pump & Port of selection valve & Volume $(\mu \mathrm{L})$ & Flow rate $\left(\mu \mathrm{L} \cdot \mathrm{s}^{-1}\right)$ \\
\hline 1 & Aspirate carrier buffer to syringe pump & In & - & 1800 & 200 \\
\hline 2 & Aspirate arsenazo III reagent & In & 1 & 100 & 100 \\
\hline 3 & Aspirate standard/sample to holding coil & In & 2 & 10 & 10 \\
\hline 4 & Aspirate arsenazo III reagent & In & 1 & 100 & 100 \\
\hline 5 & Dispense mixture to spectrophotometer & Out & 5 & 2010 & 25 \\
\hline 7 & Aspirate $1 \mathrm{M}$ hydrochloric acid & In & 3 & 200 & 200 \\
\hline 8 & Dispense to the flow-cell for washing & Out & 5 & 2000 & 2000 \\
\hline 9 & Aspirate carrier buffer to syringe pump & In & - & 1000 & 200 \\
\hline 10 & Aspirate subsequence standard/sample (rinsing step) & In & 2 & 100 & 100 \\
\hline 11 & Dispense to waste & Out & 6 & 1100 & 200 \\
\hline
\end{tabular}

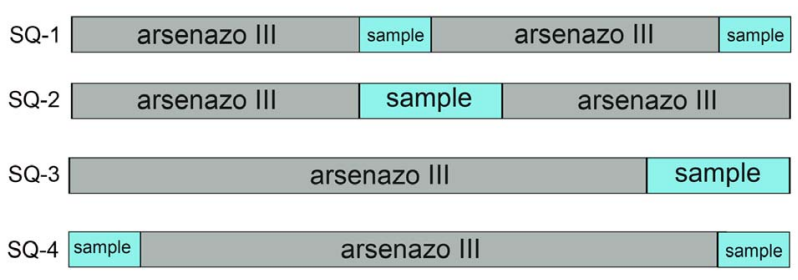

(a)

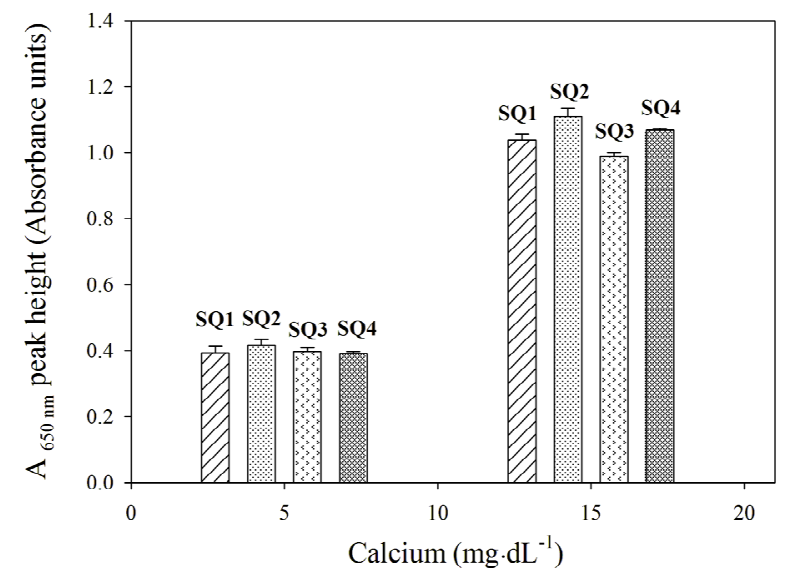

(b)

Figure 2. (a) Aspiration sequences of the arsenazo III reagent $(\mu \mathrm{L})$ and sample $(\mu \mathrm{L})$; SQ-1 (100-5-100-5 $\mu \mathrm{L})$; SQ-2 (100-10-100 $\mu \mathrm{L})$; SQ-3 (200-10 $\mu \mathrm{L})$; SQ-4 (5-200-5 $\mu \mathrm{L})$; (b) Relationship between each operation sequence versus the assay signal as assessed by 5 and $15 \mathrm{mg} \cdot \mathrm{dL}^{-1}$ calcium.

\subsubsection{Sample Volume}

To study the effect of sample volumes, the arsenazo III reagent was fixed at $200 \mu \mathrm{L}$, in which $100 \mu \mathrm{L}$ was aspirated while sandwiched between various sample volumes ranging from 5 to $75 \mu \mathrm{L}$. As shown in Figure 3, the results demonstrated that the peak height signal increased with an increase in sample volume. However, when tested with high calcium concentration $\left(15 \mathrm{mg} \cdot \mathrm{dL}^{-1}\right)$, the increase in sample volume did not obviously influence the analytical signals. Peak height curve reached a plateau while injecting a volume higher than $10 \mu \mathrm{L}$. In addition, the use of $10 \mu \mathrm{L}$ sample volume gave a wider linear range than that of other volumes. Therefore, the sample volume of $10 \mu \mathrm{L}$ was chosen for further experiments.

\subsubsection{Effect of Arsenazo III Concentration}

Taking into account of different variables in determination of calcium, the reagent concentration is one of the most significant factors in calcium assay. In this study, the operation sequence of aspirating the arsenazo III re- agent $(100 \mu \mathrm{L})$, sample $(10 \mu \mathrm{L})$, and arsenazo III reagent $(100 \mu \mathrm{L})$ was used as a model to study the effect of arsenazo III concentration. The effect of increasing arsenazo III concentration on absorbance sensitivity was investigated in the range of $50-400 \mu \mathrm{M}$. As shown in Figure 4, the analytical signals of the four different concentrations of arsenazo III were compared. The results demonstrated that the sensitivity increased with an increasing arsenazo III concentration. Hence, an arsenazo III concentration of $400 \mu \mathrm{M}$ was selected for all subsequent experiments because it provided a wider linearity, and up to $15 \mathrm{mg} \cdot \mathrm{dL}^{-1}$ of calcium could be assayed. The normal reference range for total calcium in adults is approximately 8.6 to $10.3 \mathrm{mg} \cdot \mathrm{dL}^{-1}$ [2]. The proposed assay is therefore able to detect the concentration of total calcium covering the entire clinical range.

\subsubsection{Carrier Buffer and Flow Rate}

At an alkaline $\mathrm{pH}$, arsenazo III reacts with magnesium, a divalent cation found in blood, thus reducing the specificity 


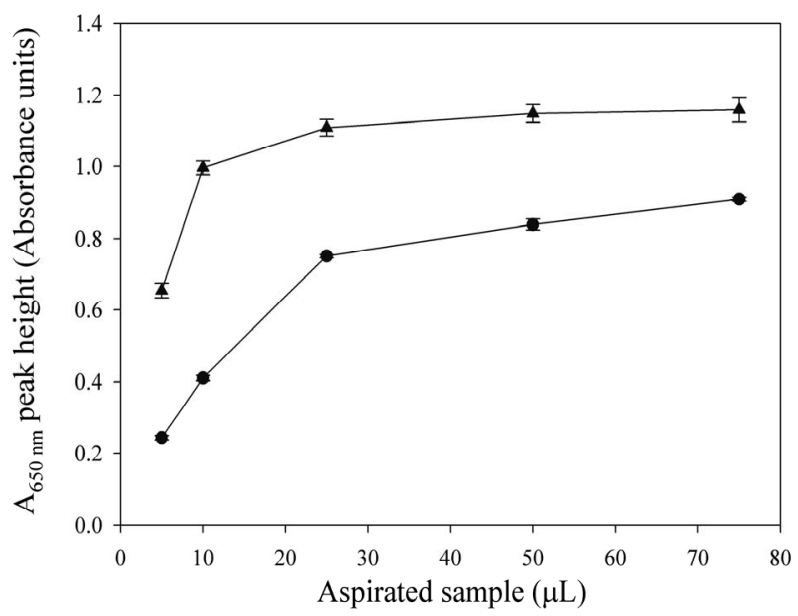

Figure 3. The relationship between the average absorbance peak height and the sample volume aspirated to the SIA system. $0,5 \mathrm{mg} \cdot \mathrm{dL}^{-1}$ calcium; $\Delta, 15 \mathrm{mg} \cdot \mathrm{dL}^{-1}$ calcium.

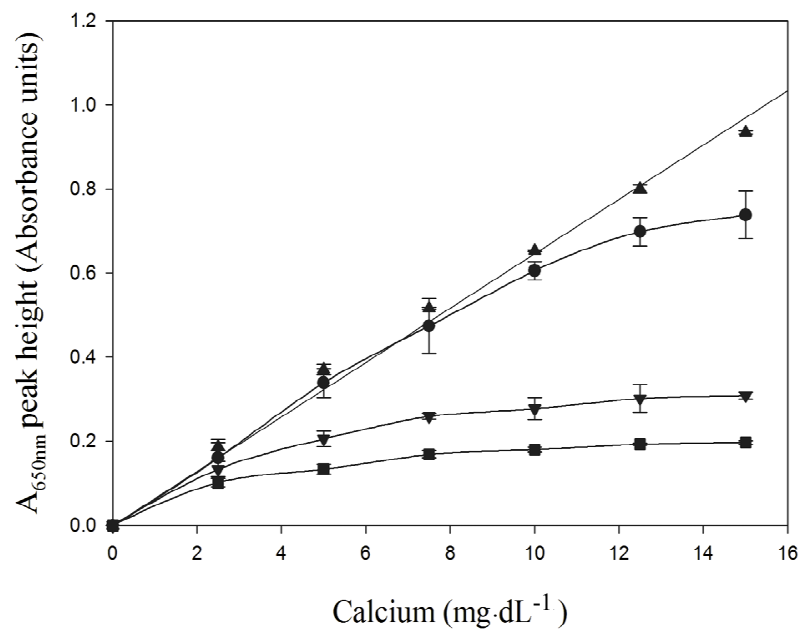

Figure 4. The effect of arsenazo III concentrations on the response signals: (匹) $50 \mu \mathrm{M},(\nabla) 100 \mu \mathrm{M},(\bullet) 200 \mu \mathrm{M}$, and ( $\triangle$ ) $400 \mu \mathrm{M}$ arsenazo III. Each concentration of calcium was assayed in duplicate and the standard deviation of assays was depicted as an error bar.

of assay performance. The hydroxyquinoline-5-sulphonic acid (8HQS) was needed as a masking agent in the alkaline reagent [17]. Although an alkaline $\mathrm{pH}$ region provides the greater sensitivity than acidic region, we decided on an acidic $\mathrm{pH}$ as a reagent to avoid the use of 8 HQS. In acidic range, it still maintains the adequate sensitivity to determine serum calcium and provides a wide linearity covering the reference interval and pathological range. Thus, the effect of sample dilution upon the assay is almost negligible.

In the preliminary studies, two types of buffers, PIPES and MES, were investigated at a $\mathrm{pH}$ of 6.5 . The results demonstrated that the use of $25 \mathrm{mM}$ PIPES buffer gave better assay signals than using $25 \mathrm{mM}$ MES buffer at the same $\mathrm{pH}$ (data not shown). Therefore, PIPES buffer was the buffer of choice for our study. In addition, three different concentrations of PIPES were also studied with 3 levels of standard calcium, which were 5, 10, and 15 $\mathrm{mg} \cdot \mathrm{dL}^{-1}$. As shown in Figure 5, the increasing concentration of PIPES provided the decrease in assay signal. The highest sensitivity was obtained with $25 \mathrm{mM}$ PIPES buffer; therefore, this concentration was chosen for further experiments.

Furthermore, the study on flow rates of the carrier buffer was under investigation for absorbance sensitivity. After completing in dye binding reaction, the calciumarsenazo III complex was dispersed to spectrophotometric detector with the constant flow rates ranging from 15 $\mu \mathrm{L} \cdot \mathrm{s}^{-1}$ to $35 \mu \mathrm{L} \cdot \mathrm{s}^{-1}$. According to Figure 6, the effect of five different flow rates on absorbance signals was compared. Considering at a steady flow rate of $25 \mu \mathrm{L} \cdot \mathrm{s}^{-1}$, the results displayed that it provided the highest response signal, thereby being selected for further experiments.

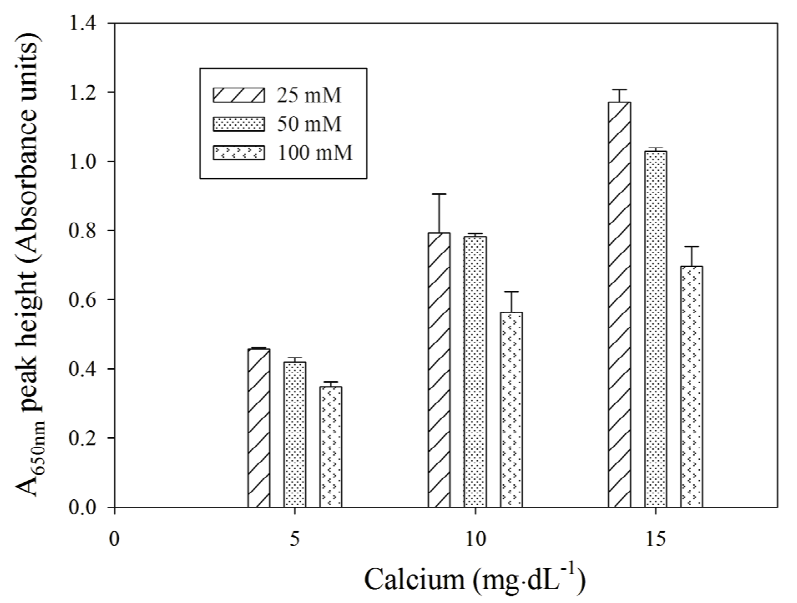

Figure 5. The effect of concentrations of PIPES buffer (25, $50,100 \mathrm{mM}$ ) on the absorbance peak height.

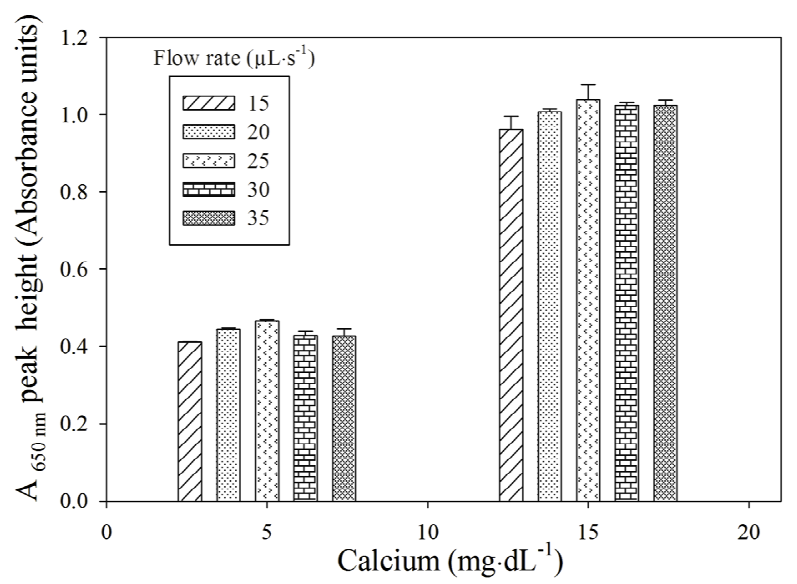

Figure 6. The effect of dispensing flow rates on the absorbance peak height. 


\subsection{Analytical Characteristics}

\subsubsection{Analytical Curve and Limit of Detection}

The absorbance peak height of the calcium standards and a typical analytical curve are shown in Figure 7(a). Linear relationships between the absorbance peak heights and concentrations of calcium were observed from 2.5 to $15 \mathrm{mg} \cdot \mathrm{dL}^{-1}$ with correlation coefficients of 0.999 , as shown in Figure 7(b). The detection limit assessed by the signal-to-noise ratio of 3 was $0.430 \mathrm{mg} \cdot \mathrm{dL}^{-1}$, or 0.107 $\mathrm{mM}$. This implies that the sensitivity of our proposed SIA system is slightly more sensitive than the method described previously by J. Nyman et al. [15]. Based on the o-cresolphthalein complexone-based SIA approach, their detection limit is maximised at $0.125 \mathrm{mM}$. However, the SIA method described here is less sensitive than our previous microfluidic-based technique [5]. Nevertheless, the SIA method was more advantageous than the microfluidic platform, in terms of high throughput analysis.

Although there have been several reports describing the versatility of the SIA system for analysis of environmental calcium in water and food $[15,16,18,19]$, until now, very few papers have exploited the SIA system for calcium determination in real clinical samples. Staden et al. [14] proposed the SIA system for determination of calcium in water, urine, and pharmaceutical samples based on the o-cresolphthalein complexone method. However, its analytical curve was linear between 0 and 2 $\mathrm{mg} \cdot \mathrm{dL}^{-1}$, which is narrower than our SIA system by a factor of approximately 7.5. Our proposed SIA system covers the broad linear range, thereby eliminating the need to dilute serum samples prior to analysis.

\subsubsection{Precision, Reproducibility, and Sample Frequency}

The reproducibility of the method was investigated with 20 runs of $10 \mathrm{mg} \cdot \mathrm{dL}^{-1}$ and $15 \mathrm{mg} \cdot \mathrm{dL}^{-1}$ calcium on the same day. The results demonstrated that within-run reproducibility was observed at $1.3 \%$ and $1.2 \% \mathrm{CVs}$ for 10 $\mathrm{mg} \cdot \mathrm{dL}^{-1}$ and $15 \mathrm{mg} \cdot \mathrm{dL}^{-1}$ calcium, respectively. Furthermore, the results of four analytical curves constructed on different consecutive days revealed good reproducibility, in which within-run CVs from $0.7 \%-1.7 \%$ and run-torun $\mathrm{CVs}$ of $2.6 \%$ were obtained.

According to the high-throughput, our proposed SIA system could process up to 30 runs per hour.

\subsubsection{Carry-Over Effect}

The carry-over effect between consecutive samples was determined by first analysing a sample containing a high analyte concentration $\left(15 \mathrm{mg} \cdot \mathrm{dL}^{-1}\right.$ calcium $)$ in triplicate, followed by analysing a sample with a low analyte concentration $\left(2.5 \mathrm{mg} \cdot \mathrm{dL}^{-1}\right.$ calcium $)$ in triplicate. The carryover effect was investigated following a method described by Haeckel et al. [20].

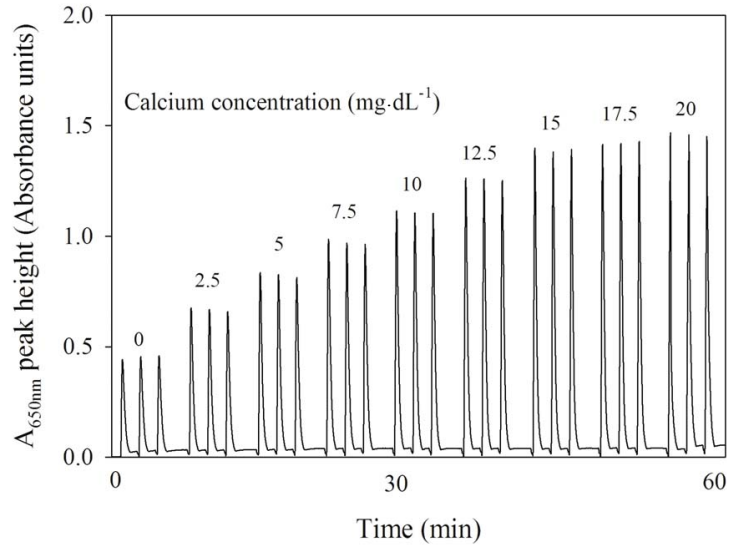

(a)

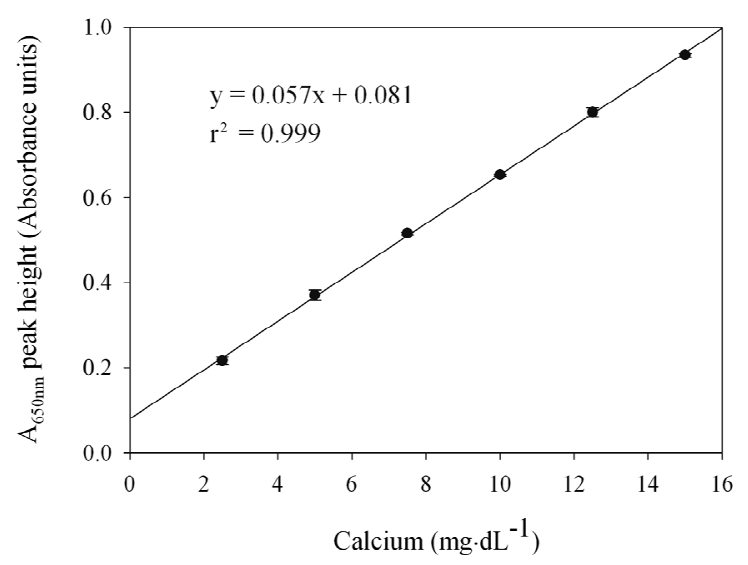

(b)

Figure 7. (a) Typical absorbance peak heights of calcium standards; (b) Analytical curve for the determination of calcium based on the proposed SIA arsenazo III method (linear range $2.5-15 \mathrm{mg} / \mathrm{dL} ; \mathrm{r}^{2}=0.999$ ).

With our method, the carry-over effect was calculated to be $0.69 \%$, which is negligible. Because the acid wash step was included in the running protocol, a low carryover effect between run was achieved.

\subsubsection{Interferences}

The interference effects on the determination of calcium from foreign substances that commonly exist in blood samples were investigated. With the aim of studying assay specificity, calcium recovery tests were carried out in the presence of other substances. Control serums (calcium $\sim 9.8 \mathrm{mg} \cdot \mathrm{dL}^{-1}$ ) containing specified concentrations of each substance were injected into the SIA system, and the absorbance peak heights were compared with those obtained from the presence of control serum alone. As shown in Table 2, the results implied that the interferences from common components in serum such as magnesium $\left(123 \mathrm{mg} \cdot \mathrm{dL}^{-1}\right)$, glucose $\left(1000 \mathrm{mg} \cdot \mathrm{dL}^{-1}\right)$, haemoglobin $\left(50 \mathrm{mg} \cdot \mathrm{dL}^{-1}\right)$, and bilirubin $\left(0.5 \mathrm{mg} \cdot \mathrm{dL}^{-1}\right)$ were acceptable, with recoveries between $99.1 \%$ - 104.4\%. 
Table 2. The influence of various tested substances on the determination of calcium by the proposed SIA system.

\begin{tabular}{ccc}
\hline Tested interferences & Added concentration $\left(\mathrm{mg} \cdot \mathrm{dL}^{-1}\right)$ & $\%$ Recovery \\
\hline None & - & 100 \\
Magnesium & 123 & 104.4 \\
Haemoglobin & 50 & 99.1 \\
Bilirubin & 0.5 & 101.3 \\
Glucose & 1,000 & 99.7 \\
\hline
\end{tabular}

\subsubsection{Method Comparison}

Clotted blood samples were centrifuged at $1500 \mathrm{~g}$ for 15 min in order to separate the serum. In our experiment, several levels of assay samples, including those with lower and higher than normal calcium levels, were obtained by spiking the real serum samples with various levels of calcium from the commercial control serums. Thirty samples were subjected to the arsenazo III SIA system for determination of calcium and compared with the results of current large-scale spectrophotometric methods. In practically, it was not necessary to dilute the actual samples from patients with saline prior to analysis, because of the rather broad linearity of our proposed system, which ranges from 2.5 to $15 \mathrm{mg} \cdot \mathrm{dL}^{-1}$ of calcium. A scatter plot of the results obtained from both methods is shown in Figure $8\left(r^{2}=0.970, n=30\right)$.

Ideally, the $y$-intercept and the slope of regression equation should be close to 0 and 1.0, respectively, for two methods with well correlation. In our current study, regression statistics were calculated to evaluate signifycant slope and intercept of regression equation, and displayed as $\mathrm{y}=(0.979 \pm 0.033) \mathrm{x}+(0.192 \pm 0.299)$. The intercept was 0.192 , with lower and upper confidence limits of -0.421 and +0.805 , respectively. This range

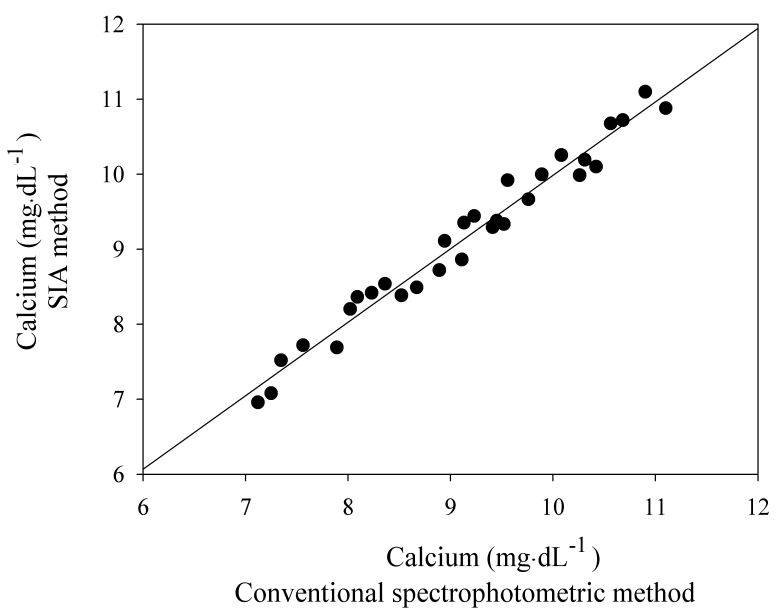

Figure 8. The proposed SIA system based on the arsenazo III method was compared to the conventional method. The regression equation was $y=0.979( \pm 0.033) x+0.192( \pm 0.299)$ and $\mathbf{r}^{2}=0.970$. included the ideal value of zero, indicating that the intercept was not significantly different from 0 at the 0.05 alpha level $(\mathrm{p}$ value $=0.526)$. The slope of graph was 0.979 with a $95 \%$ confidence interval of $0.913-1.045$, suggesting that the slope was not significantly different from 1 because this range included the model value of 1.0. With $95 \%$ confidence interval and degree of freedom of 28 , the critical $\mathrm{t}$-value $\left(\mathrm{t}_{0.05,28}\right)$ was calculated to be \pm 2.048 . Paired sample statistical analysis revealed that the experimental value of $|t|$ was 0.092 , a significantly lower than the critical t-value so the null hypothesis assumed no differences in means between the results of the two methods. These data suggest that the arsenazo II SIA system highly correlates with the conventional spectrophotometric method that utilises an assay kit from Randox ( $\mathrm{p}$-value $<0.05$ ).

\section{Conclusion}

A simple spectrophotometric SIA system for rapid determination of serum calcium has been implemented. Major advantages of the proposed system include lower arsenazo III reagent consumption and a relative throughput of 30 runs per hour. The proven low carry-over effect and the high correlation to the conventional method demonstrate the applicability of the SIA system for real serum sample analysis. In other words, the proposed SIA system has become the method of choice for clinical routine assay because it is easy to perform and offers an accurate interpretation at clinically significant levels.

\section{Acknowledgements}

The authors gratefully acknowledge the financial supports from the Thailand Research Fund, the Commission on Higher Education (MRG5380170) and the Centre for Excellence in Omics-Nano Medical Technology Project Development from Chulalongkorn University.

\section{REFERENCES}

[1] J. E. Polancic, "Elactrolytes," In: M. L. Bishop, E. P. Fody and L. E. Schoeff, Eds., Clinical Chemistry: Principles, Procedures, Correlations, 5th Edition, Lippincott Williams \& Wilkins, Philadelphia, 2005, pp. 314-342.

[2] D. B. Endres and R. K. Rude, "Disorders of Bone," In: C. A. Burtis, E. R. Ashwood and D. E. Bruns, Eds., TIETZ Fundamentals of Clinical Chemistry, 6th Edition, St. Louis Saunders Elsevier, 2008, pp. 711-733.

[3] N. O. Leary, A. Pembroke and P. F. Duggan, "Single Stable Reagent (Arsenazo III) for Optically Robust Measurement of Calcium in Serum and Plasma," Clinical chemistry, Vol. 38, No. 6, 1992, p. 904.

[4] S. Shibata, K. Goto and Y. Ishiguro, "Dual-Wavelength Spectrophotometry: Part III. Determination of Arsenazo I in Arsenazo III," Analytica Chimica Acta, Vol. 62, No. 2, 
1972, pp. 305-310. doi:10.1016/0003-2670(72)80037-0

[5] Y. Boonyasit, T. Maturos, A. Sappat, A. Jomphoak, A. Tuantranont and W. Laiwattanapaisal, "Passive Micromixer Integration with a Microfluidic Chip for Calcium Assay Based on the Arsenazo III Method," BioChip Journal, Vol. 5, 2011, pp. 1-7. doi:10.1007/s13206-011-5101-8

[6] N. Malcik, J. P. Ferrance, J. P. Landers and P. Caglar, "The Performance of a Microchip-Based Fiber Optic Detection Technique for the Determination of $\mathrm{Ca}^{2+}$ Ions in Urine," Sensors and Actuators B: Chemical, Vol. 107, No. 1, 2005, pp. 24-31. doi:10.1016/j.snb.2004.09.049

[7] P. Caglar, S. A. Tuncel, N. Malcik, J. P. Landers and J. P. Ferrance, "A Microchip Sensor for Calcium Determination," Analytical and Bioanalytical Chemistry, Vol. 386, No. 5, 2006, pp. 1303-1312. doi:10.1007/s00216-006-0776-8

[8] M. Zenki, K. Minamisawa and T. Yokoyama, "Clean Analytical Methodology for the Determination of Lead with Arsenazo III by Cyclic Flow-Injection Analysis," Talanta, Vol. 68, 2005, pp. 281-286. doi:10.1016/i.talanta.2005.07.059

[9] D. B. Gladilovich, V. Kub and L. Sommer, "Determination of the Sum of Rare-Earth Elements by Flow-Injection Analysis with Arsenazo III, 4-(2-pyridylazo) Resorcinol, Chrome Azurol S and 5-Bromo-2-(2-pyridylazo)5-diethylaminophenol Spectrophotometric Reagents," Talanta, Vol. 35, No. 4, 1988, pp. 259-265. doi:10.1016/0039-9140(88)80082-1

[10] K. Grudpan, W. Praditweangkum, P. Sooksamiti and R. Edwards, "Flow-Injection Spectrophotometric Determination of Yttrium with Arsenazo III," Laboratory Robotics and Automation, Vol. 11, 1999, pp. 279-283. doi:10.1002/(SICI) 1098-2728(1999)11:5<279::AID-LRA 6>3.0.CO;2-Y

[11] A. Rius, M. P. Callao and F. X. Rius, "Self-Configuration of Sequential Injection Analytical Systems," Analytica Chimica Acta, Vol. 316, No. 1, 1995, pp. 27-37. doi:10.1016/0003-2670(95)00346-2

[12] I. Ruisánchez, J. Lozano, M. S. Larrechi, F. X. Rius and J. Zupan, "On-Line Automated Analytical Signal Diagnosis in Sequential Injection Analysis Systems Using Artificial Neural Networks," Analytica Chimica Acta, Vol. 348, No.
1, 1997, pp. 113-127. doi:10.1016/S0003-2670(97)00147-5

[13] D. Šatínský, I. Neto, P. Solich, H. Sklenářová, M. Conceição, B. S. M. Montenegro and A. N. Araújo, "Sequential Injection Chromatographic Determination of Paracetamol, Caffeine, and Acetylsalicylic Acid in Pharmaceutical Tablets," Journal of Separation Science, Vol. 27, 2004, pp. 529-536. doi:10.1002/jssc.200301644

[14] J. F. Van Staden and R. E. Taljaard, "Determination of Calcium in Water, Urine and Pharmaceutical Samples by Sequential Injection Analysis," Analytica Chimica Acta, Vol. 323, No. 1, 1996, pp. 75-85. doi:10.1016/0003-2670(95)00615-X

[15] J. Nyman and A. Ivaska, "Spectrophotometric Determination of Calcium in Paper Machine White Water by Sequential Injection Analysis," Analytica Chimica Acta, Vol. 308, No. 1-3, 1995, pp. 286-292. doi:10.1016/0003-2670(94)00476-3

[16] J. Nyman and A. Ivasca, "Potentiometric and Spectrophotometric Determination of Calcium in the Wet End of Paper Machines by Flow Injection Analysis," Talanta, Vol. 40, No. 1, 1993, pp. 95-99. doi:10.1016/0039-9140(93)80146-I

[17] B. R. Morgan, J. D. Artiss and B. Zak, "Calcium Determination in Serum with Stable Alkaline Arsenazo III and Triglyceride Clearing," Clinical Chemistry, Vol. 39, No. 8, 1993, pp. 1608-1612.

[18] A. N. Ara jo, R. C. C. Costa, J. L. F. C. Lima and B. F. Reis, "Sequential Injection System in Flame Atomic Absorption Spectrometry for the Determination of Calcium and Magnesium in Mineral Waters," Analytica Chimica Acta, Vol. 358, No. 2, 1998, pp. 111-119. doi:10.1016/S0003-2670(97)00583-7

[19] V. Cerda, A. Cerda, A. Cladera, M. T. Oms, F. Mas, E. Gomez, F. Bauza, M. Miro, R. Forteza and J. M. Estela, "Monitoring of Environmental Parameters by Sequential Injection Analysis," TrAC Trends in Analytical Chemistry, Vol. 20, No. 8, 2001, pp. 407-418. doi:10.1016/S0165-9936(01)00064-4

[20] R. Haeckel, "Proposals for the Description and Measurement of Carry-Over Effects in Clinical Chemistry," Pure and Applied Chemistry, Vol. 63, No. 2, 1991, pp. 301-306. doi:10.1351/pac199163020301 